\title{
OBSERVABILITY-ENHANCED PROPORTIONAL NAVIGATION GUIDANCE WITH BEARINGS-ONLY MEASUREMENTS
}

\author{
MINGYAN LI ${ }^{1}$ and CHENG-CHEW LIM ${ }^{1}$
}

(Received 1 November 1997; revised 16 January 1998)

\begin{abstract}
We analyse the performance of the additive observable proportional navigation guidance system, which is well-suited for low-cost homing missiles with bearings-only measurements. Closed-form solutions are derived for both manoeuvring and non-manoeuvring targets. Guidelines on how to select the navigation constants of the control law are presented. We show that the additive observable proportional navigation guidance system can cover a larger capture area than can a conventional proportional navigation system.
\end{abstract}

\section{Introduction}

In short-range homing missiles, the proportional navigation guidance law is the most widely used $[4,5]$. This law generates a command acceleration proportional to the line-of-sight angle rate in an effort to turn the missile in a direction to reduce the line-of-sight angle rate to zero and to form a collision course. To achieve effective target interception, good sensor measurements are needed of the range rate and the line-of-sight angle rate. This may not always be possible when only a passive sensor is used to track the target or when range information from an active sensor is jammed by an intelligent target. The sensor measurement available in this case will give the bearing angle only. The realization of the guidance law will then require estimation and filtering of the range rate and the line-of-sight angle rate. This is achieved usually by an extended Kalman filter.

The state-estimation errors can however become large, especially towards the end of the missile-target interception. This is undesirable since accurate control action is essential to accomplish the mission. Investigation $[2,7]$ reveals that when the missile and the target are on a collision course, the information content of the bearingsonly measurements may not be sufficient to excite the filter under the proportional

\footnotetext{
${ }^{1}$ Department of Electrical and Electronic Engineering, The University of Adelaide, SA 5005, Australia. (C) Australian Mathematical Society 1999, Serial-fee code 0334-2700/99
} 
navigation strategy of nullifying the line-of-sight angle rate. It follows that a more effective guidance system should aim not only to nullify the angle rate, but also to enhance the information content of the measurements in order to offer the filter sufficient information to generate consistent estimates.

In selecting an information measure for enhancing filter performance, a suitable candidate is the Fisher information matrix which is a commonly-used measure of accuracy in determining unknown parameters from a sequence of measurements [8]. The matrix can be related to the missile-target intercept problem through the local observability matrix when the measurement is subject to Gaussian white noise [7]. As it is easier to handle a scalar quality in mathematical derivation and practical implementation, the trace or the determinant of the information matrix is used instead. Indeed, Speyer et al. [7] and Hull et al. [3] use the trace of the matrix as the performance index to derive numerous LQ-based guidance-control laws. The LQ-based guidance control is effective but fairly complex to implement.

A simple control scheme using the scalar measure based on the trace of the observability matrix in conjunction with a widely-used proportional navigation control law was first proposed in [2]. This scheme is motivated by meeting the two design aims of (i) retaining the simple design and implementation feature of conventional proportional navigation and (ii) offering better observability in the homing phase of the mission. The guidance law is referred to as the additive observable proportional navigation (AOPN) control law. Simulation studies given in [1] show that the control enhances the observability of the system in both non-manoeuvring and manoeuvring target engagements and helps in overcoming the filter divergence problem. Rudimentary guidelines are presented in [2] and [1] on how to select the navigation constant of the added term. It is clear that the AOPN guidance control is well-suited for lowcost homing missiles with bearings-only measurements, although refinement on the guidelines to ensure effective interception and further investigation into the effect of the new term on the capture area must be carried out.

This paper has three objectives. First, we derive closed-form solutions of the missile-target engagement under AOPN guidance for both manoeuvring and nonmanoeuvring targets. We compare these results with those based on a conventional proportional navigation law, known as the true proportional navigation (TPN) law, derived in [11]. Secondly, we show how to select the two navigation constants $N_{1}$ and $N_{2}$, where $N_{1}$ corresponds to the conventional proportional navigation part while $N_{2}$ is associated with an additive term, in order to form a collision course. The constraints are tighter than previous results in [1] and are particularly useful as design aids. Thirdly, we demonstrate that there exists an optimal value for $N_{2}$, with which the capture area of the engagement covers the largest region, and interception appears to be achieved in minimum time. Furthermore, we show that the AOPN guidance system can cover a larger capture area than can a TPN system. Computer simulation 
is used to illustrate some essential features of the control scheme and to confirm the findings.

\section{System model}

Consider the geometry of a guidance engagement shown in Figure 1, where a missile with a constant velocity $V_{M}$ attempts to intercept a target with a constant forward velocity $V_{T}$.

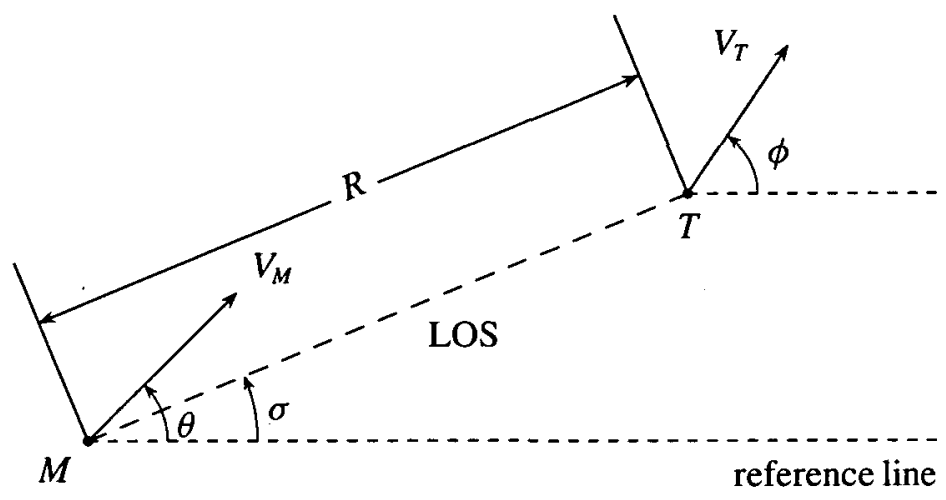

FIGURE 1. Two-dimensional guidance engagement with the missile heading at angle $\theta$ and the target heading at angle $\phi$ to the reference line.

The geometry engagement model can be written in terms of the relative distance between the missile and the target and the rate of line-of-sight (LOS) angle [4], as

$$
\begin{aligned}
\ddot{R}-R \dot{\sigma}^{2} & =0, \\
R \ddot{\sigma}+2 \dot{R} \dot{\sigma} & =-A_{n},
\end{aligned}
$$

where $\sigma$ is the LOS angle and $R$ is the relative range. Here $A_{n}$ is the commanded normal acceleration issued by the control law.

TPN is the most popular guidance law [9]. The control law is written as

$$
A_{n}=-N_{1} \dot{R} \dot{\sigma}
$$

where $N_{1}$ is the navigation constant.

However, TPN is not satisfactory for homing missiles with bearings-only measurements at the final pursuit due to the poor observability of the relative range and the range rate caused by the attempt of the law to nullify the LOS angle rate [7]. To 
enhance the observability of the system, an alternative control method, known as the additive observable proportional navigation (AOPN) was proposed in [2]. This control law augments TPN with an information-enhanced term which is closely linked to the Fisher information matrix [7, 8]. It takes the form

$$
A_{n}=-N_{1} \dot{R} \dot{\sigma}-k \dot{R} \dot{\mu},
$$

where $k$ is a constant and $\dot{\mu}$ the trace of the rate of the Fisher information matrix. Under certain fairly general assumptions [1], $\dot{\mu}$ can be simplified to the form $R^{2} / a$, where $a$ represents the variance of the measurement noise. The AOPN law becomes

$$
A_{n}=-N_{1} \dot{R} \dot{\sigma}-\frac{k}{a} \dot{R} R^{2},
$$

or

$$
A_{n}=-N_{1} \dot{R} \dot{\sigma}-N_{2} \dot{R} R^{2},
$$

where $N_{2}$ is the second navigation constant. We show how $N_{1}$ and $N_{2}$ can be selected to ensure effective interception.

\section{Analysis of guidance system}

In analysing the performance of the guidance system under the AOPN law, we start the discussion with the manoeuvring target engagement problem. Closed-form solutions are derived and conditions for effective interception established. The solutions are fairly general and are directly applicable to a non-manoeuvring target which is treated as a special case of the manoeuvring target engagement.

3.1. Manoeuvring target engagement A manoeuvring target is assumed to have an acceleration $a_{T}$ normal to the LOS and has the effect of reducing the effectiveness of the missile guidance law. For simplicity of formulation, the magnitude of $a_{T}$ is assumed to be proportional to the closing speed $\dot{R}$, that is, $a_{T}=c \dot{\sigma}_{0} \dot{R}$, where $c$ is a non-negative constant of target manoeuvrer acceleration. The differential equations describing the pursuit of a manoeuvring target under the additive observable proportional navigation law can be written as

$$
\begin{aligned}
\ddot{R}-R \dot{\sigma}^{2} & =0, \\
R \ddot{\sigma}+2 \dot{R} \dot{\sigma} & =N_{1} \dot{R} \dot{\sigma}+N_{2} \dot{R} R^{2}-a_{T}, \\
a_{T} & =c \dot{\sigma}_{0} \dot{R} .
\end{aligned}
$$

The closed-form solution and the conditions for effective interception of a manoeuvring target are now derived. 
THEOREM 1. The closed-form solution of the missile guidance system in pursuing a manoeuvring target, described by (7-9)), which represent manoeuvring target engagement using the AOPN law is when $N_{1} \neq 4$ and $N_{1}>3$,

$$
\begin{aligned}
\dot{R}^{2}= & R_{0}^{2} \dot{\sigma}_{0}^{2}\left[\frac{m^{2}}{3}\left(\frac{R}{R_{0}}\right)^{6}+m n\left(\frac{R}{R_{0}}\right)^{4}+n^{2}\left(\frac{R}{R_{0}}\right)^{2}+\frac{(1-n-m)^{2}}{N_{1}-1}\left(\frac{R}{R_{0}}\right)^{2 N_{1}-2}\right. \\
& \left.+\frac{4 m(1-n-m)}{N_{1}+2}\left(\frac{R}{R_{0}}\right)^{N_{1}+2}+\frac{4 n(1-n-m)}{N_{1}}\left(\frac{R}{R_{0}}\right)^{N_{1}}\right] \\
& +\dot{R}_{0}{ }^{2}-R_{0}^{2} \dot{\sigma}_{0}^{2}\left[\frac{m^{2}}{3}+m n+n^{2}+\frac{(1-n-m)^{2}}{N_{1}-1}\right. \\
& \left.+\frac{4 m(1-n-m)}{N_{1}+2}+\frac{4 n(1-n-m)}{N_{1}}\right]
\end{aligned}
$$

when $N_{1}=4$,

$$
\begin{aligned}
\dot{R}^{2}= & R_{0}^{2} \dot{\sigma}_{0}^{2}\left\{\left(\frac{N_{2} R_{0}^{2}}{\dot{\sigma}_{0}}\right)^{2}\left[\frac{1}{3}\left(\frac{R}{R_{0}}\right)^{6} \ln ^{2}\left(\frac{R}{R_{0}}\right)-\frac{1}{9}\left(\frac{R}{R_{0}}\right)^{6} \ln \left(\frac{R}{R_{0}}\right)+\frac{1}{54}\left(\frac{R}{R_{0}}\right)^{6}\right]\right. \\
& +\frac{2 N_{2} R_{0}^{2}(1-n)}{\dot{\sigma}_{0}}\left[\frac{1}{3}\left(\frac{R}{R_{0}}\right)^{6} \ln \left(\frac{R}{R_{0}}\right)-\frac{1}{18}\left(\frac{R}{R_{0}}\right)^{6}\right] \\
& +\frac{N_{2} R_{0}^{2} n}{\dot{\sigma}_{0}}\left[\left(\frac{R}{R_{0}}\right)^{4} \ln \left(\frac{R}{R_{0}}\right)-\frac{1}{4}\left(\frac{R}{R_{0}}\right)^{4}\right] \\
& \left.+\frac{(1-n)^{2}}{3}\left(\frac{R}{R_{0}}\right)^{6}+n(1-n)\left(\frac{R}{R_{0}}\right)^{4}+n^{2}\left(\frac{R}{R_{0}}\right)^{2}\right\}+\dot{R}_{0}^{2} \\
& -R_{0}^{2} \dot{\sigma}_{0}^{2}\left[\frac{1}{54}\left(\frac{N_{2} R_{0}^{2}}{\dot{\sigma}_{0}}\right)^{2}-\frac{5 n+4}{36}\left(\frac{N_{2} R_{0}^{2}}{\dot{\sigma}_{0}}\right)+\frac{(1-n)^{2}}{3}+n\right]
\end{aligned}
$$

where

$$
m=\frac{N_{2} R_{0}^{2}}{\left(4-N_{1}\right) \dot{\sigma}_{0}}, \quad n=\frac{c}{N_{1}-2} .
$$

ProOF. Multiplying (8) by $R / \dot{R}$ and using the unit mass angular momentum of the missile (defined by $R^{2} \sigma$ ) and initial conditions, we obtain 
when $N_{1} \neq 4$ and $N_{1}>3$,

$$
\begin{aligned}
\dot{\sigma}= & \dot{\sigma}_{0}\left(\frac{R}{R_{0}}\right)^{N_{1}-2}+\frac{N_{2}}{4-N_{1}} R^{2}\left[1-\left(\frac{R}{R_{0}}\right)^{N_{1}-4}\right] \\
& +\frac{c \dot{\sigma}_{0}}{N_{1}-2}\left[1-\left(\frac{R}{R_{0}}\right)^{N_{1}-2}\right]
\end{aligned}
$$

when $N_{1}=4$,

$$
\dot{\sigma}=\dot{\sigma}_{0}\left(\frac{R}{R_{0}}\right)^{2}+N_{2} R^{2} \ln \left(\frac{R}{R_{0}}\right)+\frac{c \dot{\sigma}_{0}}{N_{1}-2}\left[1-\left(\frac{R}{R_{0}}\right)^{2}\right] .
$$

The LOS angle rate $\dot{\sigma}$ given in (13) and (14) is obtained also by imposing the constraint $N_{1}>3$ which is shown to be necessary for effective interception in Theorem 2 . Substituting (13) and (14) into (7) and using $\ddot{R} d R=d\left(\dot{R}^{2} / 2\right)$, the solution of (7) and (8) can be written as (10) and (11).

\section{REMARKS.}

- As $\lim _{R \rightarrow 0} R^{2} \ln \left(R / R_{0}\right)=0$, it can be concluded from (13) and (14) that the LOS angle rate $\dot{\sigma}$ will approach $c \dot{\sigma}_{0} /\left(N_{1}-2\right)$ as long as the range approaches zero towards the end of the interception for both $N_{1} \neq 4$ and $N_{1}=4$.

- The final LOS angular rate is proportional to the target manoeuvrer constant $c$. This means that the angular rate will increase with the increase of the target manoeuvrer constant.

- The expression for $\dot{\sigma}$ consists of three parts. The first is caused by TPN, the second is due to the additive term to enhance observability and the last part is due to the target manoeuvrer.

- The results are equally applicable to TPN guidance, with $N_{2}=0$.

THEOREM 2. For effective interception of a manoeuvring target, the following constraints must be satisfied when using the AOPN guidance law given by (6):

$$
\begin{aligned}
& N_{1}>3, \\
& A^{2}>\frac{1}{N_{1}+2}+\frac{\left(c^{2}-4 c\right) N_{1}+3 c^{2}}{4 N_{1}^{2}\left(N_{1}+2\right)}, \\
& N_{2}<\frac{\dot{\sigma}_{0}}{2 R_{0}^{2} N_{1}}\left[3\left(c N_{1}+2 N_{1}+c\right)+b\right],
\end{aligned}
$$

where $A=\frac{\dot{R}_{0}}{R_{0} \dot{\sigma}_{0}}$,

$$
b=\sqrt{3\left(N_{1}-1\right)\left[4 A^{2} N_{1}^{3}+\left(8 A^{2}-4\right) N_{1}^{2}+\left(4 c-c^{2}\right) N_{1}-3 c^{2}\right]} .
$$


PROOF. To guarantee effective capture, the missile should intercept the target with a finite acceleration and within a finite time [10]. From (13) and (14), $N_{1}$ should be larger than 2 to prevent $\dot{\sigma}$ from becoming infinity when $R$ approaches zero at the final course of pursuit. Substituting $\dot{\sigma}$ into (8) with (13), $\ddot{\sigma}$ can be written as

$$
\begin{aligned}
\ddot{\sigma}= & \dot{R}\left\{( N _ { 1 } - 2 ) \left[\dot{\sigma_{0}} \frac{R^{N_{1}-3}}{R_{0}^{N_{1}-2}}+\frac{N_{2}}{4-N_{1}}\left(R-\frac{R^{N_{1}-3}}{R_{0}^{N_{1}-4}}\right)\right.\right. \\
& \left.\left.+\frac{c \dot{\sigma}_{0}}{N_{1}-2}\left(1-\frac{R^{N_{1}-3}}{R_{0}^{N_{1}-2}}\right)\right]+N_{2} R-\frac{c \dot{\sigma}_{0}}{R}\right\} .
\end{aligned}
$$

Equation (19) shows that $N_{1}>3$ can prevent the LOS angular acceleration $\ddot{\sigma}$ from approaching infinity, as $R \rightarrow 0$. The final closing speed $\dot{R}_{f}$ can be derived from (10) and (11) as

$$
\begin{aligned}
\frac{\dot{R}_{f}}{\dot{R}_{0}}= & \left\{1-\frac{1}{A^{2}}\left[\frac{m^{2}}{3}+m n+n^{2}+\frac{(1-n-m)^{2}}{N_{1}-1}\right.\right. \\
& \left.\left.+\frac{4 m(1-n-m)}{N_{1}+2}+\frac{4 n(1-n-m)}{N_{1}}\right]\right\}^{\frac{1}{2}} .
\end{aligned}
$$

In order to obtain a real $\dot{R}_{f}$, the following constraint must be satisfied:

$$
\begin{aligned}
A^{2}> & \frac{m^{2}}{3}+m n+n^{2}+\frac{(1-n-m)^{2}}{N_{1}-1}+\frac{4 m(1-n-m)}{N_{1}+2} \\
& +\frac{4 n(1-n-m)}{N_{1}} .
\end{aligned}
$$

The inequality (21) determines the ranges of $\dot{\sigma}_{0}, R_{0}$ and $\dot{R}_{0}$. If these initial conditions with a given $N_{1}$ and $N_{2}$ cannot satisfy the inequality, interception (or capture) will not be achieved. Thus the inequality defines the capture area. Rewriting (21) with $m, n$ given in (12), as

$$
\begin{aligned}
0> & {\left[\left(\frac{R_{0}{ }^{2}}{\dot{\sigma}_{0}}\right)^{2} N_{1}\right] N_{2}^{2}-\left\{\frac{3 R_{0}^{2}}{\dot{\sigma}_{0}}\left[(c+2) N_{1}+c\right]\right\} N_{2} } \\
& +3\left(N_{1}+2\right)\left[N_{1}+(c+2) c-N_{1}\left(N_{1}-1\right) A^{2}\right]
\end{aligned}
$$

and solving the inequality, the upper bound of $N_{2}$ in (17) is obtained. If and only if $b$, given by (18), is real, then the bound of $N_{2}$ is real. We have

$$
\left(N_{1}-1\right)\left\{4 N_{1}^{2}\left(N_{1}+2\right) A^{2}-\left[4 N_{1}^{2}+\left(c^{2}-4 c\right) N_{1}+3 c^{2}\right]\right\}>0 .
$$

Note that $N_{1}>1$ because of (15). Therefore, (23) leads to the bound of $A^{2}$ in (16). 


\section{REMARKS.}

- The constraints (15-17), are necessary conditions for effective capture.

- (15) and (17) provide guidelines for choosing $N_{1}$ and $N_{2}$.

THEOREM 3. $N_{2}$ of the AOPN guidance law has an optimal value $N_{20 p t}$ (in terms of the largest capture area and the fastest final closing speed)

$$
N_{2 \mathrm{opt}}=\frac{3 \dot{\sigma}_{0}}{2 R_{0}^{2}}\left(c+2+\frac{c}{N_{1}}\right) \text {. }
$$

PROOF. Rearranging the inequality (21) and replacing $m$ and $n$ with (12), we have

$$
A^{2}>\frac{\left[\left(\frac{R_{0}^{2}}{\dot{\sigma}_{0}}\right)^{2} N_{1}\right] N_{2}^{2}-\left\{\frac{3 R_{0}^{2}}{\dot{\sigma}_{0}}\left[(c+2) N_{1}+c\right]\right\} N_{2}+3\left(N_{1}+2\right)\left(N_{1}+2 c+c^{2}\right)}{3 N_{1}\left(N_{1}+2\right)\left(N_{1}-1\right)} .
$$

When $N_{2}=N_{2 o p t}$, the lower bound of $A^{2}$ is found to be the minimum, that is,

$$
\frac{1}{N_{1}+2}+\frac{\left(c^{2}-4 c\right) N_{1}+3 c^{2}}{4 N_{1}^{2}\left(N_{1}+2\right)}
$$

This means that the system has the largest capture area for a given $N_{1}$ and a given target manoeuvrer acceleration $c$. At the same time, the absolute final closing speed $\dot{R}_{f}$ in (20) reaches its maximum given by

$$
\left|\dot{R}_{f}\right|_{\max }=\left|\dot{R}_{0}\right| \sqrt{1-\frac{1}{A^{2}}\left[\frac{1}{N_{1}+2}+\frac{\left(c^{2}-4 c\right) N_{1}+3 c^{2}}{4 N_{1}^{2}\left(N_{1}+2\right)}\right]} .
$$

REMARKS. The lower bound of $A^{2}$ under TPN, that is, $N_{2}=0$ in (25), is

$$
\frac{N_{1}+2 c+c^{2}}{N_{1}\left(N_{1}-1\right)}
$$

and under AOPN, it can reach

$$
\frac{4 N_{1}^{2}+\left(c^{2}-4 c\right) N_{1}+3 c^{2}}{4 N_{1}^{2}\left(N_{1}+2\right)} .
$$

The bound due to the AOPN is smaller by

$$
\frac{3\left[(c+2) N_{1}+c\right]^{2}}{4 N_{1}^{2}\left(N_{1}-1\right)\left(N_{1}+2\right)} \text {. }
$$

Since the smaller the lower bound of $A^{2}$, the larger the capture area will be, the missile-target system under AOPN with the optimal value of $N_{2}$ has a larger capture area than that under TPN. 
3.2. Non-manoeuvring target engagement The results obtained in the previous section are fairly general and are directly applicable to a non-manoeuvring target engagement as a special case of manoeuvring target engagement with $c=0$ in (9). As the non-manoeuvring target engagements are extensively used in the study of guidance systems $[4,5,7,11]$, we summarize, for completeness, the results of the non-manoeuvring target engagement in the following.

The missile guidance engaging a non-manoeuvring target using the AOPN given in (6) is written as

$$
\begin{aligned}
\ddot{R}-R \dot{\sigma}^{2} & =0, \\
R \ddot{\sigma}+2 \dot{R} \dot{\sigma} & =N_{1} \dot{R} \dot{\sigma}+N_{2} \dot{R} R^{2} .
\end{aligned}
$$

THEOREM 4. The closed-form solution of the missile guidance system engaging a non-manoeuvring target under AOPN described by (27) and (28) is, when $N_{1} \neq 4$ and $N_{1}>3$,

$$
\begin{aligned}
\dot{R}^{2}= & R_{0}^{2} \dot{\sigma}_{0}^{2}\left[\frac{m^{2}}{3}\left(\frac{R}{R_{0}}\right)^{6}+\frac{4 m(1-m)}{N_{1}+2}\left(\frac{R}{R_{0}}\right)^{N_{1}+2}+\frac{(1-m)^{2}}{N_{1}-1}\left(\frac{R}{R_{0}}\right)^{2 N_{\mathrm{t}}-2}\right] \\
& +\dot{R}_{0}^{2}-R_{0}^{2} \dot{\sigma}_{0}^{2}\left[\frac{m^{2}}{3}+\frac{4 m(1-m)}{N_{1}+2}+\frac{(1-m)^{2}}{N_{1}-1}\right]
\end{aligned}
$$

when $N_{1}=4$,

$$
\begin{aligned}
\dot{R}^{2}= & \frac{R_{0}^{2} \dot{\sigma}^{2}}{3}\left(\frac{R}{R_{0}}\right)^{6}\left\{1+\left(\frac{N_{2} R_{0}^{2}}{\dot{\sigma}_{0}}\right)^{2}\left[\ln ^{2}\left(\frac{R}{R_{0}}\right)-\frac{1}{3} \ln \left(\frac{R}{R_{0}}\right)+\frac{1}{18}\right]\right. \\
& \left.+\frac{2 N_{2} R_{0}^{2}}{\dot{\sigma}_{0}}\left[\ln \left(\frac{R}{R_{0}}\right)-\frac{1}{6}\right]\right\}+\dot{R}_{0}^{2} \\
& -\frac{R_{0}^{2} \dot{\sigma}_{0}^{2}}{3}\left[1-\frac{N_{2} R_{0}^{2}}{3 \dot{\sigma}_{0}}+\frac{1}{18}\left(\frac{N_{2} R_{0}^{2}}{\dot{\sigma}_{0}}\right)^{2}\right],
\end{aligned}
$$

where $m$ is given in (12).

The LOS angular rate $\dot{\sigma}$ can be obtained from (13) and (14) as, when $N_{1} \neq 4$ and $N_{1}>3$,

$$
\dot{\sigma}=\dot{\sigma}_{0}\left(\frac{R}{R_{0}}\right)^{N_{1}-2}+\frac{N_{2}}{\left(4-N_{1}\right)} R^{2}\left[1-\left(\frac{R}{R_{0}}\right)^{N_{1}-4}\right] ;
$$

when $N_{1}=4$,

$$
\dot{\sigma}=\dot{\sigma}_{0}\left(R / R_{0}\right)^{2}+N_{2} R^{2} \ln \left(R / R_{0}\right)
$$


REMARKS.

- The LOS angular rate engaged in a non-manoeuvring target will approach zero.

- The response of the range rate given by (29) and (30) can be divided into two parts. The first term is linked to the pursuit motion and the other is determined by the system's initial conditions and the navigation constants.

THEOREM 5. For effective interception of a non-manoeuvring target, the constraints that must be satisfied when the AOPN guidance law is used are

$$
\begin{gathered}
N_{1}>3, \\
A^{2}>\frac{1}{N_{1}+2}, \\
\frac{\dot{\sigma}_{0}}{R_{0}^{2}}(3+d)>N_{2} \geq 0 \text { for } A^{2}>\frac{1}{N_{1}-1}, \\
\frac{\dot{\sigma}_{0}}{R_{0}^{2}}(3+d)>N_{2}>\frac{\dot{\sigma}_{0}}{R_{0}^{2}}(3-d) \text { for } \frac{1}{N_{1}-1} \geq A^{2}>\frac{1}{N_{1}+2},
\end{gathered}
$$

where

$$
d=\sqrt{9-3\left(N_{1}+2\right)\left(1+A^{2}-N_{1} A^{2}\right)}
$$

REMARKS.

- Since $c=0$ simplifies the expression of condition (21) for effective interception, both the lower bound and upper bound of $N_{2}$ can be obtained in (35).

- Comparing (17) with (35), the upper bound of $N_{2}$ for a manoeuvring target is larger than that for a non-manoeuvring target. This is because greater missile manoeuvrability is required when the target is manoeuvring.

- Comparing (16) with (34), the lower bound for $A^{2}$ involved in manoeuvring target engagement given by (16) can be separated into two parts. The first is equal to that of a non-manoeuvring target and the second is caused by the target manoeuvrer.

- When $N_{1}>3$ and $0<c<2$, the lower bound of $A^{2}$ of the manoeuvring target case (16) is less than that of a non-manoeuvring target (34). This means that for a missile to intercept a manoeuvring target under AOPN, a larger capture area than that of a non-manoeuvring target is more likely.

- AOPN has a major advantage over TPN. Under TPN the capture area decreases with an increase in target manoeuvrability [11], while under AOPN the capture area in the presence of a target manoeuvrer will be larger than that of a nonmanoeuvring case, provided $N_{1}>3$ and $0<c<2$. 
THEOREM 6. $N_{2}$ of the AOPN guidance law for non-manoeuvring target engagement has an optimal value (in terms of the largest capture area and the fastest final closing speed)

$$
N_{\text {2opt }}=3 \dot{\sigma}_{0} / R_{0}^{2}
$$

\section{REMARKS.}

- When $N_{2}=N_{2 o p t}$, the lower bound of $A^{2}$ reaches its minimum, that is, $1 /\left(N_{1}+2\right)$. This means that the system has the largest capture area for a given $N_{1}$.

- When $N_{\text {2opt }}$ is used, the absolute final closing speed becomes

$$
\left|\dot{R}_{f}\right|_{\max }=\left|\dot{R}_{0}\right| \sqrt{1-\frac{1}{A^{2}\left(N_{1}+2\right)}},
$$

which is the maximum.

- The maximum absolute final speed for a non-manoeuvring target given by (38) is not necessarily smaller than that for a manoeuvring target given by (26). It depends on the value of $c$ and $N_{1}$.

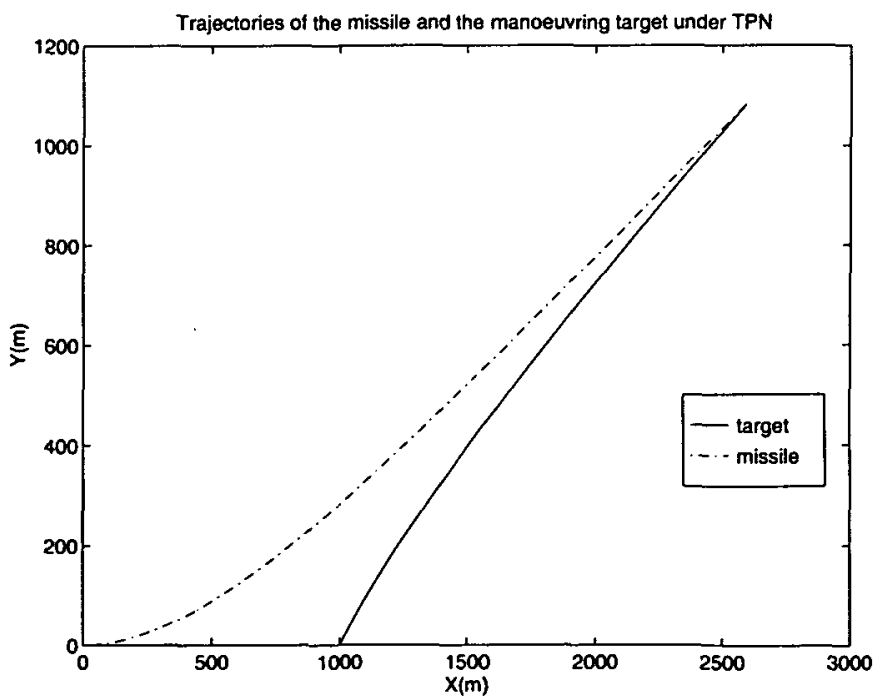

FIGURE 2. Missile and target trajectories using TPN for $N_{\mathrm{l}}=4$ and $c=1$; target initial acceleration $a_{T 0}=8.2 \mathrm{~g}$. 


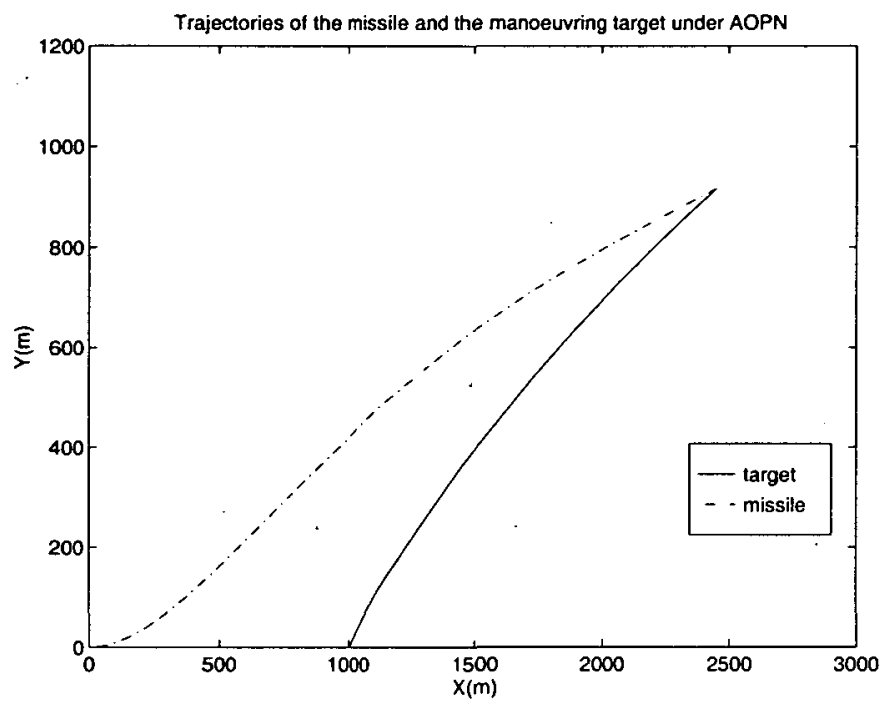

FIGURE 3. Missile and target trajectories using AOPN for $N_{1}=4, N_{2}=N_{20 p t}$ and $c=1$; target initial acceleration $a_{T 0}=8.2 \mathrm{~g}$.

\section{Simulation and discussion}

In order to evaluate the performance of the AOPN law and to confirm the results derived in Section 3, simulation studies with different scenarios are conducted.

All the simulations use the following data:

velocity of the missile, $V_{M}=600 \mathrm{~m} / \mathrm{s}$;

velocity of the target, $V_{T}=300 \mathrm{~m} / \mathrm{s}$;

initial heading angle of the missile, $\theta_{0}=0^{\circ}$;

initial heading angle of the target, $\phi_{0}=45^{\circ}$;

initial LOS angle, $\sigma_{0}=0^{\circ}$;

initial relative range, $R_{0}=1000 \mathrm{~m}$.

The initial conditions $\dot{R}_{0}$ and $\dot{\sigma}_{0}$ are computed by

$$
\begin{aligned}
& \dot{\sigma}_{0}=\frac{V_{T} \sin \left(\phi_{0}-\sigma_{0}\right)-V_{M} \sin \left(\theta_{0}-\sigma_{0}\right)}{R_{0}}, \\
& \dot{R}_{0}=V_{T} \cos \left(\phi_{0}-\sigma_{0}\right)-V_{M} \cos \left(\theta_{0}-\sigma_{0}\right) .
\end{aligned}
$$

Figure 2 shows the trajectories of the missile and the manoeuvring target using the TPN law, while Figure 3 shows the trajectories under the AOPN guidance with $N_{2 \text { opt }}$. Comparing with that under TPN, the missile guided by the AOPN law swings its way during the pursuit course so as to obtain more information about the relative range 


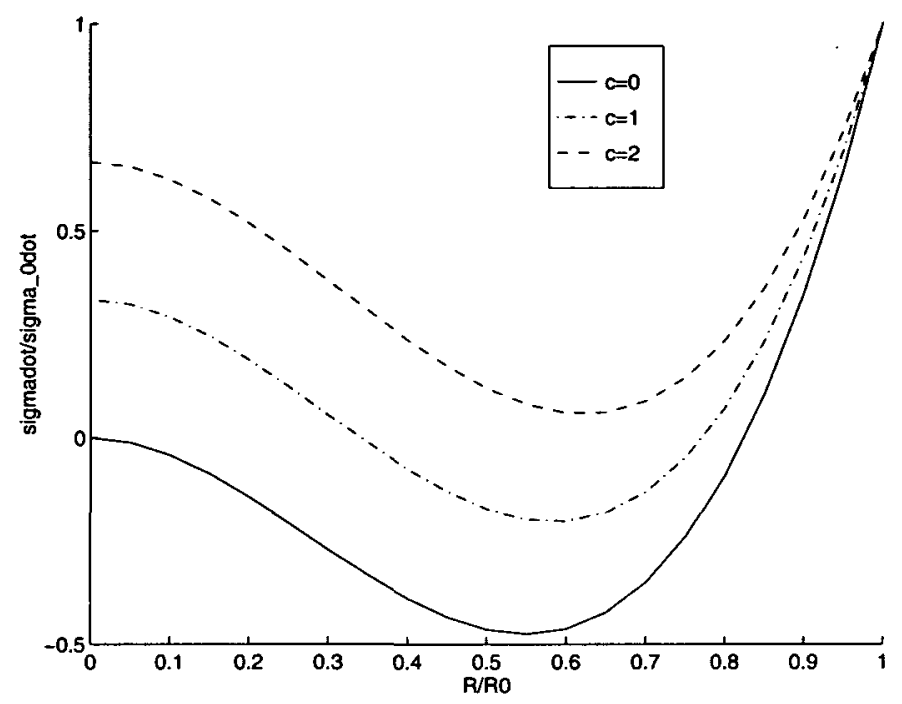

FIGURE 4. $\frac{\dot{\sigma}}{\dot{\sigma}_{0}}$ vs $\frac{R}{R_{0}}$ with $N_{1}=5, N_{2}=1 \times 10^{-6}$ and three different $c$ values.

TABLE 1. Optimal values and upper bounds of $N_{2}$ with different $c$ values when $N_{1}=4$

\begin{tabular}{||c|c|c||}
\hline $\mathbf{c}$ & $N_{\text {2opt }}\left(10^{-6}\right)$ & $N_{\text {2upb }}\left(10^{-6}\right)$ \\
\hline \hline $\mathbf{0}$ & 0.63 & 3.39 \\
\hline $\mathbf{1}$ & 1.03 & 3.80 \\
\hline $\mathbf{2}$ & 1.42 & 4.18 \\
\hline
\end{tabular}

and the range rate. In this way, the aim to enhance the observability of the system can be fulfilled.

Figure 4 is derived from (13). It shows that the LOS angular rate $\dot{\sigma}$ approaches zero for the non-manoeuvring target (that is, $c=0$ ) towards the end of pursuit, that is, when $R / R_{0} \rightarrow 0$. For the manoeuvring target, the final angular rate increases with the increase in target manoeuvrability, as discussed in Theorem 2.

Using (10), we obtain Figure 5, which confirms that the final range rate, which occurred at $R / R_{0}=0$, is the largest with optimal $N_{2}$ as discussed in Theorem 3 .

The capture areas which are defined by the constraints (15), (16) and (17) are plotted in Figure 6. It demonstrates that the system with $N_{2 o p t}$ has the largest capture area. Note that the smaller the lower bound of $A^{2}$, the larger the capture area ( $c f$. (16)).

The upper bounds of $\mathrm{N}_{2}$ and the optimal values of $\mathrm{N}_{2}$ with different target manoeuvrer acceleration $c$ are compared in Table 1, where $N_{2 \text { upb }}$ denotes the upper bound of $N_{2}$. When $N_{2}=N_{2 o p t}$, the duration of pursuit is usually the shortest (see Table 2). 


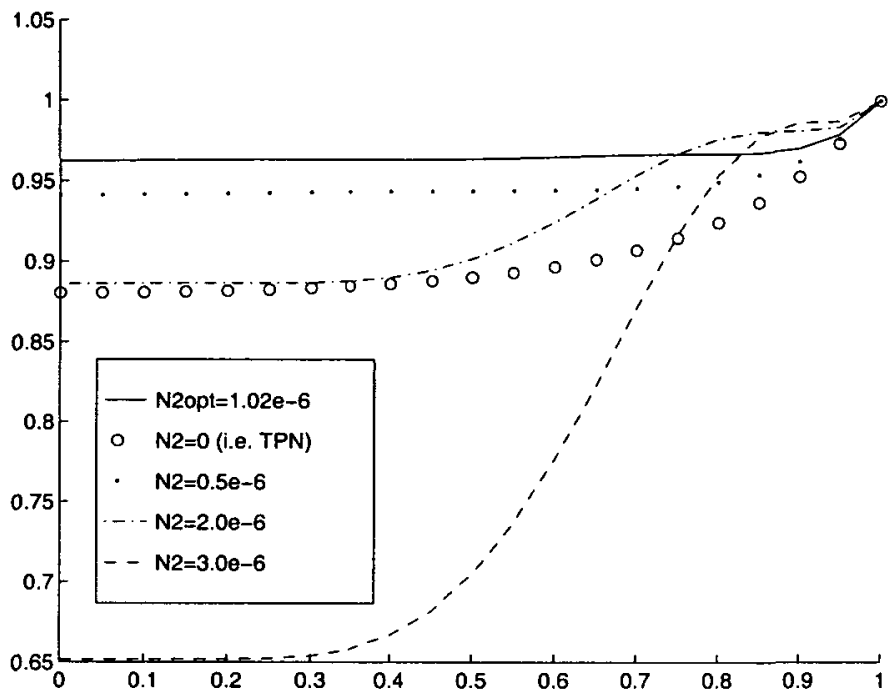

FIGURE 5. $\dot{R} / \dot{R}_{0}$ vs $R / R_{0}$ with $N_{1}=5, c=1$ and five different $N_{2}$ values.

TABLE 2. Duration of the pursuit with different values of $c$ and $N_{2}$.

\begin{tabular}{||c|c|c|c||}
\hline \multirow{2}{*}{$\mathbf{c}$} & \multicolumn{2}{|c|}{ AOPN } & TPN \\
\cline { 2 - 4 } & $N_{\text {2opt }}$ & $N_{2}=2.2 \times 10^{-6}$ & $N_{2}=0$ \\
\hline \hline $\mathbf{0}$ & $2.28(\mathrm{~s})$ & $2.94(\mathrm{~s})$ & $2.34(\mathrm{~s})$ \\
\hline $\mathbf{1}$ & $2.61(\mathrm{~s})$ & $2.78(\mathrm{~s})$ & $2.76(\mathrm{~s})$ \\
\hline $\mathbf{2}$ & $2.62(\mathrm{~s})$ & $2.69(\mathrm{~s})$ & $2.89(\mathrm{~s})$ \\
\hline
\end{tabular}

From the simulations above, it is suggested that the AOPN guidance performs better than TPN when $A^{2}$ is small. A small value of $A^{2}$ represents a small initial range rate, a large initial distance, or a large initial LOS angular rate. In other words, the missile is under less favorable initial engagement conditions. Under these circumstances, the AOPN law with $N_{2 o p t}$ has a larger capture area and a shorter pursuit duration than those under TPN.

\section{Conclusion}

In this paper we present an analytical study of the additive observable proportional navigation for a low-cost homing missile. The exact and complete closed-form solutions to the AOPN guidance problem are derived for manoeuvring target engagement with non-manoeuvring target engagement as a special case. Guidelines for choosing navigation constants are proposed. Some important characteristics related to system 


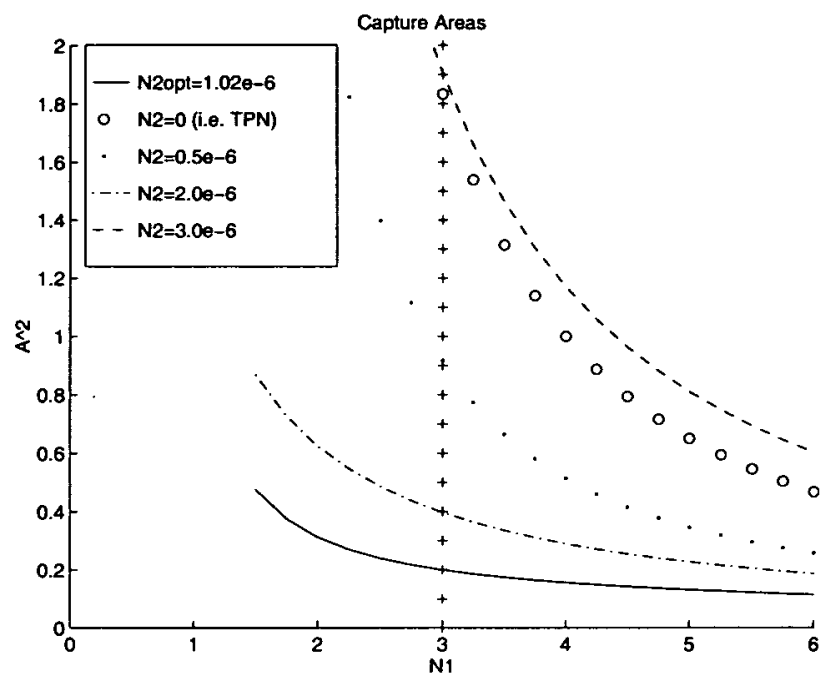

FIGURE 6. Capture area for five different $N_{2}$ values when $c=1$. Note that $N_{1}$ must be larger than 3 , and that a small $A^{2}$ represents less favorable initial engagement conditions needed to achieve interception.

performance are discussed in detail. Our investigation shows that there exists an optimal $\mathrm{N}_{2}$ in terms of the largest capture area and the fastest final closing speed which usually leads to the least duration of interception. AOPN demonstrates better performance than TPN by covering a larger capture area.

\section{Acknowledgments}

The work of Mingyan Li has been supported by an OPRS award and a University of Adelaide Scholarship.

\section{References}

[1] G. E. Hassoun, "Study of observability-enhanced guidance system", Ph. D. Thesis, The University of Adelaide, October 1995.

[2] G. E. Hassoun and C. C. Lim, "Advanced guidance control system design for homing missiles with bearings-only measurement", in IEEE International Conference on Industrial Technology, (Guangzhou, China, December, 1994), 250-254.

[3] D. G. Hull, J. L. Speyer and D. B. Burris, "Linear-quadratic guidance law for dual control of homing missiles", J. of Guidance. Control and Dynamics 13 (1990) 137-144.

[4] S. A. Murtaugh and H. E. Criel, "Fundamentals of proportional navigation", IEEE Spectrum December (1966) 75-85. 
[5] H. L. Patrick, S. M. Seltzer and M. E. Warren, "Guidance laws for short-range tactical missiles", J. of Guidance and Control 4 (1981) 98-108.

[6] J. L. Speyer, D. G. Hull and W. P. Bernard, "Performance of the modified-gain extended Kalman filter along an enhanced information path of a homing missile", in Proceedings of the AIAA Guidance and Control Conference (Williamsburg, Virginia, August 1986), 48-56.

[7] J. L. Speyer, D. G. Hull and C. Y. Tseng, "Estimation enhancement by trajectory modulation for homing missiles", J. of Guidance, Control and Dynamics 7 (1984) 167-174.

[8] B. L. van der Waerden, Mathematical Statistics (George Allen and Unwin Ltd., London, 1969).

[9] C. D. Yang, H. B. Hsiao and F. B. Yeh, "Generalized guidance law for homing missiles", IEEE Transactions on Aerospace and Electronic Systems 25 (1989) 197-211.

[10] C. D. Yang and F. B. Yeh, "Closed-form solution for a class of guidance law", J. of Guidance, Control and Dynamics 10 (1987) 412-415.

[11] P. J. Yuan and J. S. Chern, "Solutions of true proportional navigation for maneuvering and nonmaneuvering targets", J. of Guidance, Control and Dynamics 15 (1992) 267-271. 